\title{
SET and MYND domain-containing protein 3 inhibits tumor cell sensitivity to cisplatin
}

\author{
LEI WANG $^{1,2^{*}}$, MAN-LI XU ${ }^{1,2^{*}}$, CHANG WANG $^{1,2^{*}}$, QING-QING DONG ${ }^{1,2}$, ZHI MIAO ${ }^{1,2}$, XIAO-YING CHEN $^{1,2}$, \\ NAN WANG ${ }^{1,2}$, HONG-PENG HE ${ }^{1,2}$, TONG-CUN ZHANG ${ }^{1,2}$ and XUE-GANG LUO ${ }^{1,2}$ \\ ${ }^{1}$ Key Laboratory of Industrial Fermentation Microbiology, Tianjin University of Science and Technology, \\ Ministry of Education; ${ }^{2}$ Tianjin Key Laboratory of Industrial Microbiology, College of Biotechnology, \\ Tianjin University of Science and Technology, Tianjin 300457, P.R. China
}

Received December 11, 2018; Accepted January 24, 2020

DOI: $10.3892 / \mathrm{ol} .2020 .11465$

\begin{abstract}
Cisplatin resistance has been a major factor limiting its clinical use as a chemotherapy drug. The present study aimed to investigate whether SET and MYND domain-containing protein 3 (SMYD3), a histone methyltransferase closely associated with tumors can affect the sensitivity of tumors to cisplatin chemotherapy. Real time-qPCR, western blotting, the luciferase reporter, MTT and clonogenic assays were performed to detect the effects of SMYD3 on the chemotherapy capacity of cisplatin. In the present study, SMYD3 exhibited different expression patterns in MCF-7 and T47D breast cancer cells. In addition, this differential expression was associated with tumor cell resistance to cisplatin. Furthermore, SMYD3 knockdown following small interfering RNA transfection increased cisplatin sensitivity, whereas SMYD3 overexpression decreased cisplatin sensitivity. In addition, SMYD3 knockdown synergistically enhanced cisplatin-induced cell apoptosis. SMYD3 expression was downregulated during cisplatin treatment. In addition, transcriptional regulatory activities of SMYD3 3'-untranslated region were also downregulated. These results suggested that SMYD3 may affect cell sensitivity to cisplatin and participate in the development of cisplatin resistance, which is a process that may involve microRNA-124-mediated regulation.
\end{abstract}

\section{Introduction}

Cisplatin is an important chemotherapy drug that was approved by the Food and Drug Administration 40 years ago (1). It has

Correspondence to: Professor Xue-Gang Luo, Tianjin Key Laboratory of Industrial Microbiology, College of Biotechnology, Tianjin University of Science and Technology, 29, 13th Street, Tianjin 300457, P.R. China

E-mail: luoxuegang@hotmail.com

*Contributed equally

Key words: SET and MYND domain containing 3, cisplatin sensitivity, tumor cells since been widely used in the clinical treatment of various types of solid tumor including breast, bladder, head and neck, lung, ovarian and testicular cancers (2-4). However, its use has been limited due to inherent and acquired resistance (5).

The pathways involved in the acquired cisplatin resistance are complex. The main resistance mechanisms include DNA damage repair and cell cycle interference (6), mitochondrial dysfunction (7), inhibition of cell apoptosis (8), formation of an anoxic microenvironment (9) and epithelial-mesenchymal transition (10). Numerous strategies can be used to avoid cisplatin resistance by influencing the molecular mechanisms of resistance in tumor cells. These strategies include the development of novel platinum antitumor drugs, the promotion of platinum-based drug transport into tumor cells, the development of inhibitors that regulate the mechanisms of resistance, and the synergistic use of cisplatin with other drugs presenting specific and targeted effects on tumor cells $(11,12)$.

SET and MYND domain containing 3 (SMYD3) is a protein that catalyzes the methylation of histones at $\mathrm{H} 3 \mathrm{~K} 4$, H4K5 and H4K20, and which elicits oncogenic effects by activating the transcription of downstream target genes in hepatocellular, colorectal, cervical and breast cancers (13). For example, SMYD3 can promote epithelial-mesenchymal transition (14), interfere with the cell cycle (15), promote cell proliferation (16), increase the activity of telomerase and promote cell immortalization (17). These processes are also closely associated with chemotherapy-resistance (18). In addition, SMYD3 deficiency induces DNA-damage hypersensitivity, decreases the number of repair foci and leads to impaired homologous recombination, all of which are also associated with chemotherapy resistance (19). At present, research around SMYD3 signaling has mainly focused on its downstream pathways. Studies have found that hepatitis $\mathrm{C}$ virus core proteins can inactivate miR-124 through DNA methylation, thereby up-regulating SMYD3, while miR-124 can promote cancer cell sensitivity to cisplatin (20-22). In addition, previous studies from our laboratory demonstrated that SMYD3 can increase cellular resistance to dexamethasone (23) and regulate the ATM-CHK2/p53-cdc25c pathway (15), suggesting that SMYD3 may also be involved in the development of chemotherapy resistance. However, the direct effect of cisplatin on SMYD3 and the underlying 
mechanisms are unclear. Therefore, the present study assessed these issues. Research on this topic is of vital importance, and may serve the development of novel treatment strategies that would overcome cisplatin resistance and allow the clinical treatment of tumors.

\section{Materials and methods}

Cell lines and transfection. MCF-7 and T47D human breast cancer cells (The Cell Bank of Type Culture Collection of the Chinese Academy of Sciences) were cultured in RPMI 1640 medium (Gibco; Thermo Fisher Scientific, Inc.) supplemented with $10 \%$ fetal bovine serum (Hangzhou Sijiqing Biological Engineering Materials Co., Ltd.), $100 \mathrm{U} / \mathrm{ml}$ penicillin $\mathrm{G}$ and $0.1 \mathrm{mg} / \mathrm{ml}$ streptomycin and placed at $37^{\circ} \mathrm{C}$ in a humidified incubator containing $5 \% \mathrm{CO}_{2}$. The overexpression plasmid of SMYD3 was a gift from Professor Philip Tucker (Institute for Cellular and Molecular Biology, University of Texas, Austin, TX, USA), and the empty vector was used as a control. The small interfering (si) RNAs were synthesized by Guangzhou RiboBio Co., Ltd. The sequences of these siRNAs were as follows: siRNA targeting SMYD3 (si-SMYD3), sense, 5'-CAAGGAUGCUGAUAUGCUAdTdT-3' and antisense, 3'-dTdT GUUCCUACGACUAUACGAU-5'. The sequence of the control siRNA was 5'-ACTGTTCTATGACTTGTC GTGAATA-3'. Transient transfection was performed using TurboFect reagent (Thermo Fisher Scientific, Inc.) according to the manufacturer's instructions. Briefly, $1 \mu \mathrm{g}$ plasmid or siRNA were transfected into MCF-7 or T47D cells with $2 \mu \mathrm{l}$ TurboFect reagent. Cells were collected 24 or 48 h later for subsequent testing.

Reverse transcription quantitative $(R T-q) P C R$ and semi-qPCR. Total RNA was isolated from MCF-7 and T47D cells using TRIzol reagent (Invitrogen; Thermo Fisher Scientific, Inc.) under RNase-free conditions. After quantification of RNA using a photometer, cDNA was synthesized using M-MLV reverse transcriptase (Invitrogen, Thermo Fisher Scientific, Inc.) from $2 \mu \mathrm{g}$ total RNA, according to the manufacturer's instructions.

For semi-qPCR, primers specific for the cDNAs of the SMYD3 gene and the constitutive GAPDH gene were used (Genewiz Inc.). For SMYD3, the forward primer positions were 647-670 (5'-CCCAGTATGTCTTTGCTGAATCAC-3') and the reverse primer positions were 935-956 (5'-ACTTCCAGT GCGCCTTCAGCTC-3'). For GAPDH, the forward primer positions were 217-236 (5'-ATTCAACGGCACAGTCAA GG-3') and the reverse primer positions were 411-429 (5'-GCA GAAGGGGCGGAGATGA-3'). The PCR reactions were as follows: $95^{\circ} \mathrm{C}$ for $5 \mathrm{~min}$, then 30 cycles at $95^{\circ} \mathrm{C}$ for $1 \mathrm{~min}$, at $56^{\circ} \mathrm{C}$ for $1 \mathrm{~min}$, and at $72^{\circ} \mathrm{C}$ for $30 \mathrm{sec}$; extension was carried out at $72^{\circ} \mathrm{C}$ for $10 \mathrm{~min}$. PCR products were electrophoretically separated by $1.5 \%$ agarose gels and expression observed using ethidium bromide staining. The densities of the bands were analyzed using Gel/Chemi Doc (Bio-Rad Laboratories, Inc.) and quantified using Quantity One software v 4.6.6 (Bio-Rad Laboratories, Inc.).

qPCR was performed using Fast SYBR Green Master Mix (Applied Biosystems; Thermo Fisher Scientific Inc.) and detected using an ABI Step One system (Thermo Fisher
Scientific, Inc.). The sequences of the primers (Invitrogen; Thermo Fisher Scientific, Inc.) were as follows: GAPDH forward, 5'-ATTCAACGGCACAGTCAAGG-3' and reverse, 5'-GCAGAAGGGGCGGAGATGA-3'; SMYD3 forward, 5'-CCCAGTATCTCTTTGCTCAATCAC-3' and reverse, 5'-ACTTCCAGTGTGCCTTCAGTTC-3'; miR-124 forward, 5'- ACACTCCAGCTGGGTAAGGCACGCGG-3' and reverse, 5'-TGGTGTCGTGGAGTCG-3'. qPCR was performed as follows: $95^{\circ} \mathrm{C}$ for $2 \mathrm{~min}$, followed by 40 cycles of $95^{\circ} \mathrm{C}$ for $10 \mathrm{sec}, 65^{\circ} \mathrm{C}$ for $30 \mathrm{sec}$ and $72^{\circ} \mathrm{C}$ for $30 \mathrm{sec}$. The $2^{-\Delta \Delta \mathrm{Cq}}$ method was used to calculate relative transcription levels and all experiments were repeated at least three times (24).

Western blotting. A total of $48 \mathrm{~h}$ after transfection, MCF-7 and T47D cells were washed twice with ice-cold PBS and lysed at $4^{\circ} \mathrm{C}$ with RIPA lysis buffer containing protease and phosphatase inhibitors (cat. no. R0010; Beijing Solarbio Science \& Technology Co., Ltd.) for $30 \mathrm{~min}$. The lysates were centrifuged at $17,000 \mathrm{x} \mathrm{g}$ for $10 \mathrm{~min}$, the supernatants were quantified using the BCA protein concentration assay kit (Beyotime Institute of Biotechnology) and mixed with SDS sample buffer. Subsequently, proteins (50 $\mu \mathrm{g} /$ lane) were separated using $12 \%$ SDS-PAGE and transferred onto nitrocellulose membranes. Membranes were blocked using 5\% non-fat dry milk dissolved in PBS for $90 \mathrm{~min}$ at $20^{\circ} \mathrm{C}$. Membranes were then incubated with primary antibodies against SMYD3 (rabbit anti-human monoclonal antibody; 1:1,000; cat. no. ab187149; Abcam) and GAPDH (mouse anti-human; dilution; 1:5,000; cat. no. sc-365402; Santa Cruz) at $4^{\circ} \mathrm{C}$ overnight. Subsequently, membranes were incubated with infrared fluorescent goat anti-rabbit (1:10,000; cat. no. 926-68071; LI-COR Biosciences;) and goat anti-mouse (1:10,000; cat. no. 926-32210; LI-COR Biosciences;) secondary antibodies for $2 \mathrm{~h}$ in the dark at room temperature. Signals were visualized using an Odyssey Imaging System (LI-COR Biosciences).

Luciferase reporter assay. The SMYD3 3'-untranslated region (3'UTR) was subcloned into pGL3 Basic vector (Promega Corporation). MCF-7 or T47D cells were seeded into 24 -well plates at a density of $1 \times 10^{8}$ cells $/ \mathrm{ml}$ and then treated with different concentrations of cisplatin $(0,2,4,8,16,32$ or $64 \mu \mathrm{M})$ for $24 \mathrm{~h}$ and transfected with pGL3-SMYD3 3'UTR and pEGFP-C3 plasmids using TurboFect reagent (Thermo Fisher Scientific, Inc.) according to the manufacturer's instructions. The luciferase activity was measured using the Luciferase assay system (Promega Corporation) according to the manufacturer's instructions. The values were normalized to the signal of enhanced green fluorescent protein and the $0 \mu \mathrm{M}$ cisplatin group was used as control group (25).

MTT assay. MCF-7 and T47D cells were seeded into 96-well plates at the density of $5 \times 10^{4}$ cells $/ \mathrm{ml}$. After serum-free medium starvation for $24 \mathrm{~h}$, cells were transfected with siRNA or overexpression SMYD3 and corresponding controls as aforementioned. Cells transfected with overexpression or small interference SMYD3 and co-treated with cisplatin for 24, 48 and $72 \mathrm{~h}$ were incubated with $5 \mathrm{mg} / \mathrm{ml} \mathrm{MTT} \mathrm{(Beijing}$ Solarbio Science \& Technology Co., Ltd.) at $37^{\circ} \mathrm{C}$ for $4 \mathrm{~h}$. Subsequently, medium was removed, $150 \mu \mathrm{l}$ DMSO was added in each well to dissolve formazan crystals and optical density 
A

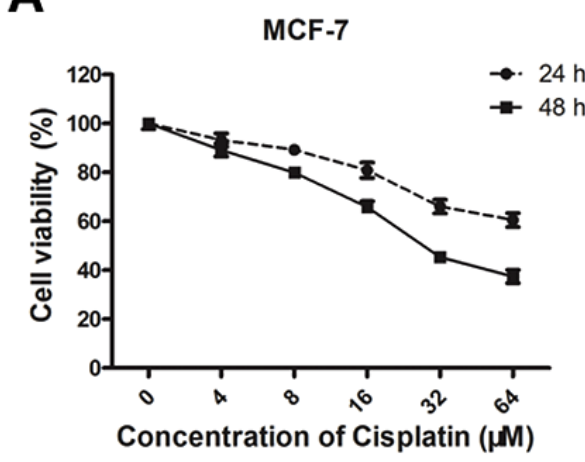

B

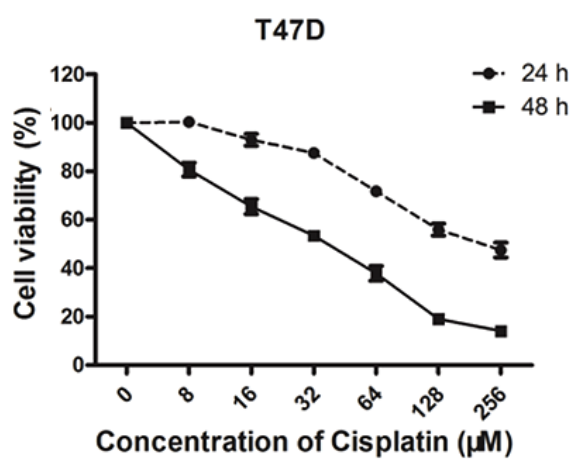

C
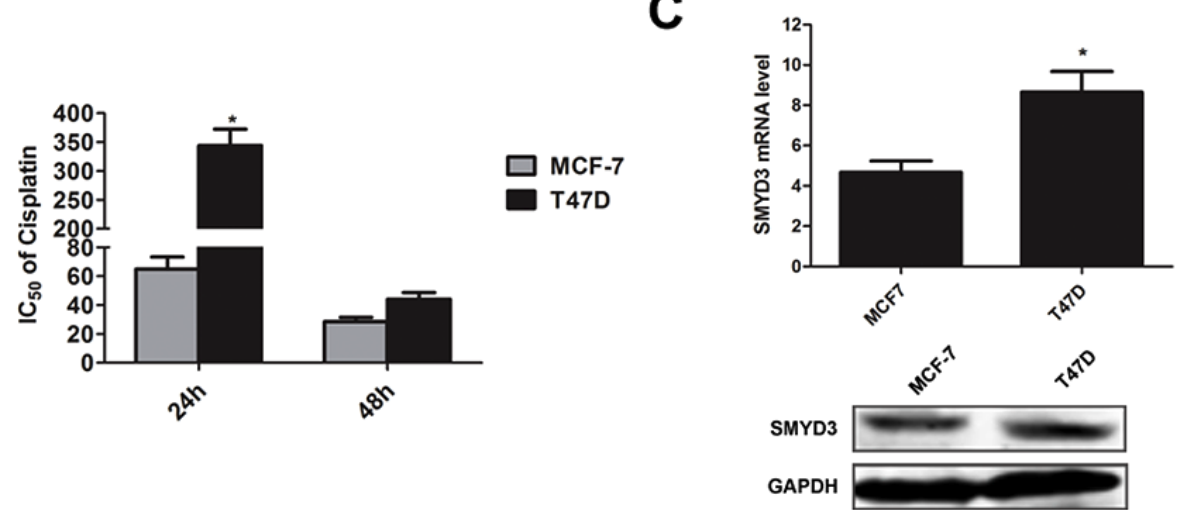

Figure 1. Cisplatin sensitivity may be inversely related to endogenous SMYD3 expression. (A) Effect of cisplatin treatment on MCF-7 and T47D cell viability after 24 or $48 \mathrm{~h}$ was determined. (B) Cisplatin $\mathrm{IC}_{50}$ in MCF-7 and T47D cell lines. (C) SMYD3 expression in MCF-7 and T47D cells assessed by reverse transcription quantitative PCR and western blotting. ${ }^{*} \mathrm{P}<0.05$ vs. MCF7 cells. SMYD3, SET and MYND domain-containing protein 3.

(OD) was measured at $570 \mathrm{~nm}$ using a Synergy4 microplate reader (BioTek Instruments, Inc.; Agilent Technologies, Inc.). Cell viability was calculated as follows: Cell viability $(\%)=[\mathrm{OD}$ (treated)-OD (blank)]/[OD (untreated)-OD (blank)] x100. Each sample was examined in duplicate, and the experiment was repeated three times.

Clonogenic assay. Following transfection with siRNA or treatment with $0,2,4,8,16$ and $32 \mu \mathrm{M}$ cisplatin at $37^{\circ} \mathrm{C}$ for $24 \mathrm{~h}$, cells were seeded in a 6-well plate at a density of 1,000 cells/well. Cells were subsequently cultured for 10 days at $37^{\circ} \mathrm{C}$ to obtain visible colonies. Cells were washed twice with PBS, fixed with methanol for $15 \mathrm{~min}$ at room temperature and stained with Giemsa for $30 \mathrm{~min}$ at room temperature. An inverted fluorescence microscope, ECLIPSE TE2000 (20x) was used to count colonies containing at least 50 cells (Nikon Corporation).

Analysis of cell mitochondrial membrane potential. Previous studies demonstrated that cisplatin can promote apoptosis by reducing mitochondrial membrane potential $(26,27)$. In the present study, MCF-7 cells were treated with 0 or $8 \mu \mathrm{M}$ cisplatin and/or siRNA targeting SMYD3 mRNA for $48 \mathrm{~h}$ at $37^{\circ} \mathrm{C}$. Then cells were harvested with trypsin and resuspended in medium at a density of $1 \times 10^{6}$ cells $/ \mathrm{ml}$. Subsequently, cells were mixed with $50 \mu \mathrm{g} / \mathrm{ml}$ rhodamine 123 dye solution (Sigma-Aldrich; Merck $\mathrm{KGaA}$ ), which is a positively charged pigment that can bind to mitochondria with a high negative membrane potential (28). After being incubated at $37^{\circ} \mathrm{C}$ and $5 \% \mathrm{CO}_{2}$ for $30 \mathrm{~min}$. Cells were collected by centrifugation at $1,700 \mathrm{x} g$ for $10 \mathrm{~min}$ at room temperature, washed twice with PBS and analyzed in the Accuri C6 flow cytometer (Becton Dickinson Biosciences, USA) using FlowJo v 10.2 (FlowJo LLC).

Statistical analysis. Data are presented as the means \pm standard deviation (mean \pm SD). Significant differences were evaluated using two-way ANOVA. Statistical analyses were performed using GraphPad Prism version 5.00 (GraphPad Software, Inc.). All experiments were carried out at least three times. $\mathrm{P}<0.05$ was considered to indicate a statistically significant difference.

\section{Results}

Cisplatin sensitivity may be inversely related to endogenous SMYD3 expression. MTT assays were performed to assess the effect of cisplatin on MCF-7 and T47D cell viability after 24 and $48 \mathrm{~h}$ treatment. As presented in Fig. 1A, following cisplatin treatment, viability of T47D cells was lower compared with MCF-7 cells. This indicates that MCF-7 cells may be more sensitive to cisplatin. Furthermore, the expression of SMYD3 in these two cell lines was further investigated by RT-qPCR and western blotting. The results demonstrated that both mRNA and protein level of SMYD3 in the T74D cells were higher compared with MCF-7 cells (Fig. 1C). To quantitatively assess the effects of cisplatin on these two cell lines, the half maximal inhibitory concentration $\left(\mathrm{IC}_{50}\right)$ values of cisplatin were calculated. As presented in Fig. 1B, following $24 \mathrm{~h}$ of cisplatin treatment, the observed $\mathrm{IC}_{50}$ values for MCF-7 and 
A
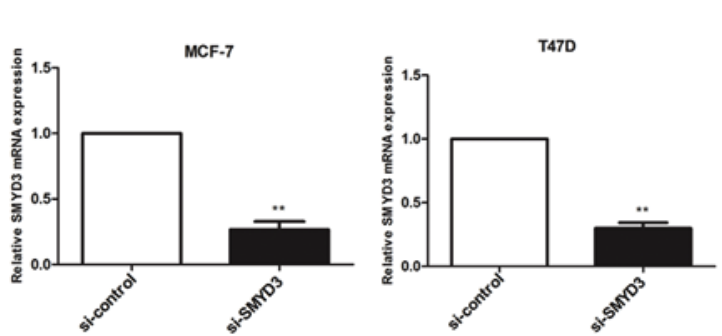

B
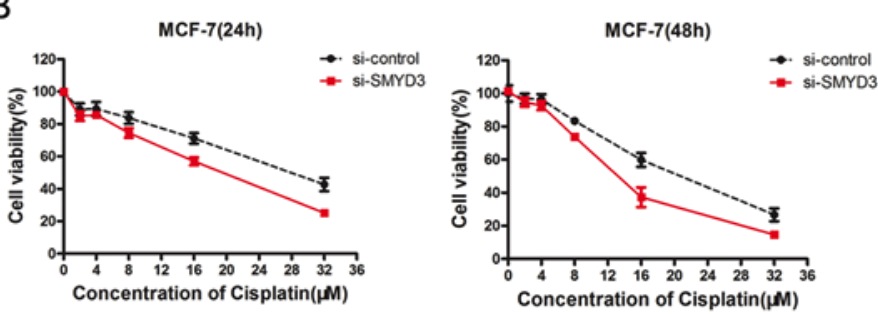

C
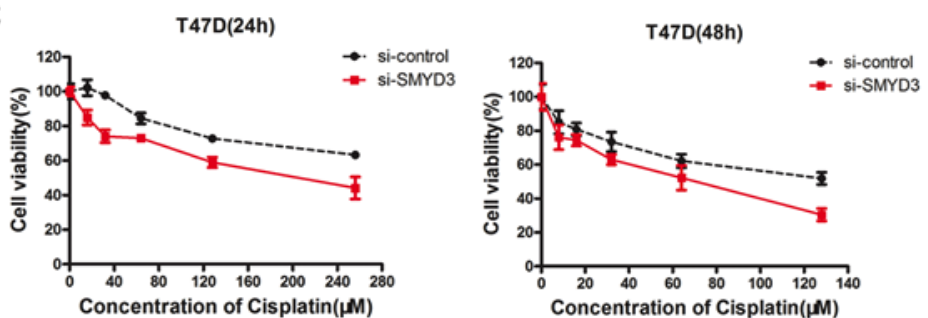

D
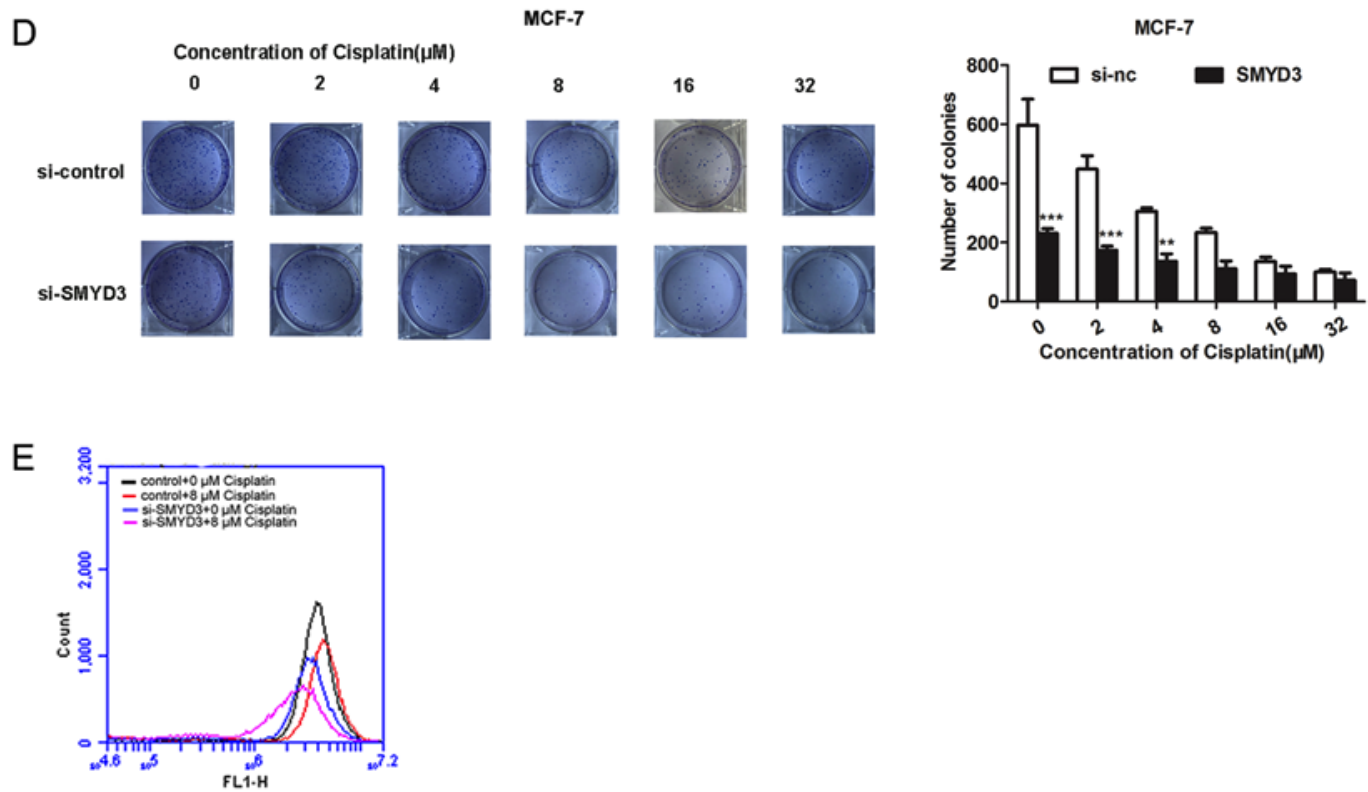

Figure 2. SMYD3 knockdown increases cisplatin sensitivity in tumor cells. (A) MCF-7 and T47D cells were transfected with si-SMYD3 or si-control before exposure to different concentrations of cisplatin. Changes in SMYD3 mRNA level were detected by reverse transcription quantitative PCR. Effect of SMYD3 knockdown on (B) MCF-7 and (C) T47D cell sensitivity to cisplatin sensitivity measured with the MTT assay and (D) colony-formation assay. (E) The membrane potential of mitochondria was assessed by Rhodamine 123 efflux assays with MCF-7 cells. Mean $\pm \mathrm{SD}$. $\mathrm{n}=3$. ${ }^{* *} \mathrm{P}<0.01,{ }^{* * * *} \mathrm{P}<0.001$ vs. si-control. si, small interfering; SMYD3, SET and MYND domain-containing protein 3; nc, negative control.

T47D cells were $62.13 \pm 3.58$ and $342.76 \pm 17.66 \mu \mathrm{M}$, respectively. These results indicated that the differential expression of SMYD3 observed may be associated with the different cisplatin sensitivities of these tumor cell lines.

SMYD3 knockdown increases cisplatin sensitivity in tumor cells. SMYD3 is often highly expressed in cancer tissues and is closely associated with malignancy and poor prognosis of patients $(29,30)$. However, the association between SMYD3 expression, chemotherapy and its outcomes remains unclear. In the present study, SMYD3 was significantly knocked down by siRNA transfection in MCF-7 and T47D cell lines (Fig. 2A), and MTT and colony-formation assays were performed to assess the cisplatin sensitivity in these two cell lines. The results demonstrated that SMYD3 knockdown significantly reduced the cell viability following cisplatin treatment (Fig. 2B and C), suggesting that SMYD3 may be involved in the development of cell resistance to cisplatin. To verify this hypothesis, further colony-formation assays were performed using MCF-7 cells (Fig. 2D) and the sensitivity of these cells to cisplatin was enhanced after SMYD3 knockdown. In addition, to investigate whether SMYD3 can affect the effect of cisplatin on the mitochondrial membrane potential of cells, rhodamine 123 staining was performed. As presented in Fig. 2E, SMYD3 knockdown decreased the mitochondrial membrane potential, which was enhanced in the presence of cisplatin. These results demonstrated that SMYD3 knockdown and cisplatin treatment may cooperatively promote the loss of the mitochondrial membrane potential in cancer cells. 
A

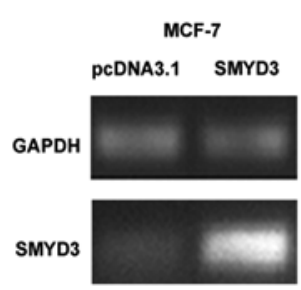

B

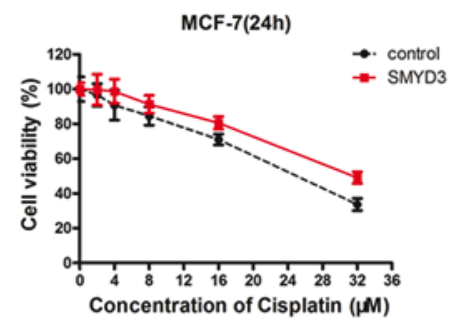

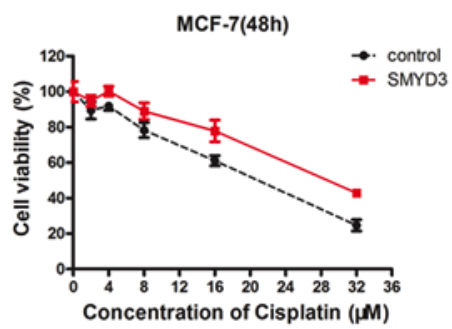

C
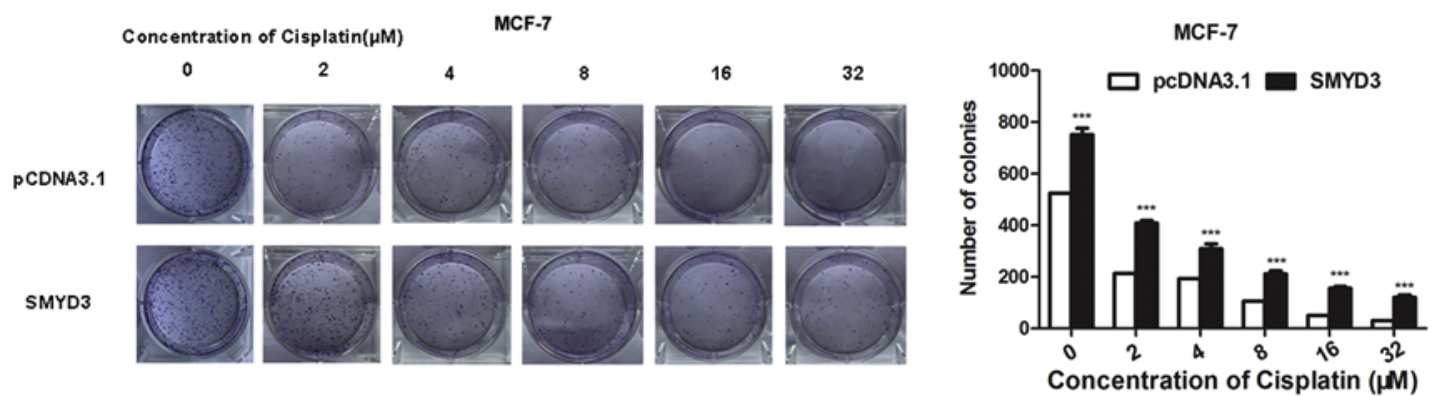

Figure 3. SMYD3 overexpression decreases cell cisplatin sensitivity. MCF-7 cells were transfected with SMYD3 overexpression plasmids and control vector (A) Semi-qPCR was performed to detect SMYD3 mRNA level, and (B) the effect of SMYD3 overexpression on cell sensitivity to cisplatin was measured with the MTT assay and $(C)$ colony-formation assay. Mean $\pm \mathrm{SD}$. $n=4 .{ }^{* * * *} \mathrm{P}<0.001 \mathrm{vs}$. pcDNA3.1. SMYD3, SET and MYND domain-containing protein 3 .

SMYD3 overexpression reduces cisplatin sensitivity. MCF-7 cells were successfully transfected with the overexpression plasmid of SMYD3 or a control empty plasmid. (Fig. 3A). Following transfection for $24 \mathrm{~h}$, SMYD3-overexpressing cells exhibited increased cell viability and increased tolerance to cisplatin compared with control (Fig. 3B). Similar results were also observed following transfection of cells with the SMYD3 overexpression plasmid for $48 \mathrm{~h}$ (Fig. 3B). The results of the colony-formation assay also demonstrated that overexpression of SMYD3 could significantly increase the tolerance of cells to cisplatin (Fig. 3C).

Cisplatin inhibits SMYD3 expression. The aforementioned results demonstrated that cell sensitivity to cisplatin may be associated with SMYD3 expression. To determine the underlying mechanisms of cisplatin-mediated cytotoxicity, MCF-7 and T47D cells were treated with cisplatin at different concentrations for 24 or $48 \mathrm{~h}$, and SMYD3 expression level was assessed by RT-PCR and western blotting. Following treatment with cisplatin for 24 and $48 \mathrm{~h}$, SMYD3 mRNA level was significantly downregulated, suggesting that cisplatin can inhibit the transcription of SMYD3 (Fig. 4A and B). These results of western blotting analysis also demonstrated that SMYD3 expression was decreased following cisplatin treatment in a concentration-dependent manner (Fig. 4C).

miR-124 may be involved in the cisplatin-mediated inhibition of SMYD3 expression. Since miRNAs can combine with the 3'UTRs of mRNAs to inhibit transcription (31). In the present study, a luciferase reporter assay was used to test whether cisplatin-mediated inhibition of SMYD3 expression may occur via the regulation of a specific miRNA that mediates SMYD3 transcription. This assay assessed the transcriptional activity of SMYD3 3'UTR following treatment with different concentrations of cisplatin (Fig. 5). Following cisplatin treatment, the luciferase activity of the SMYD3 3'UTR was significantly downregulated (Fig. 5A), suggesting that cisplatin may inhibit SMYD3 expression by upregulating the transcription of a miRNA that could promote the degradation of SMYD3 mRNA.

As previous studies reported that miR-124 is an upstream regulator of SMYD3 $(20,32)$, the present study hypothesized that miR-124 may be involved in the inhibitory effect of cisplatin on the expression of SMYD3. To verify this hypothesis, MCF-7 and T47D cells were treated for $24 \mathrm{~h}$ with different concentrations of cisplatin and the level of miR-124 was assessed by RT-qPCR. For both cell lines, miR-124 level was significantly increased following cisplatin treatment in concentration-dependent manner in MCF-7 and T47D cell lines, respectively (Fig. 5B), suggesting that cisplatin may inhibit SMYD3 expression by upregulating miR-124.

\section{Discussion}

The present study demonstrated that SMYD3 expression may affect cell sensitivity to cisplatin, SMYD3 overexpression or knockdown could decreased or enhanced the sensitivity of MCF-7 and T47D cells to cisplatin. The results demonstrated that the use of SMYD3-targeting siRNA may reduce cell resistance to cisplatin, providing therefore a novel approach to the treatment of cancer.

Cisplatin causes cytotoxicity by inducing apoptosis, which involves the induction of exogenous and endogenous death receptors through mitochondrial pathways (33). The main mechanism of cisplatin resistance involves the inactivation of apoptotic protein p53 (34). Dai et al (35) reported that SMYD3 regulates p53 protein expression, which is essential in SMYD3-induced glioma cell proliferation. 


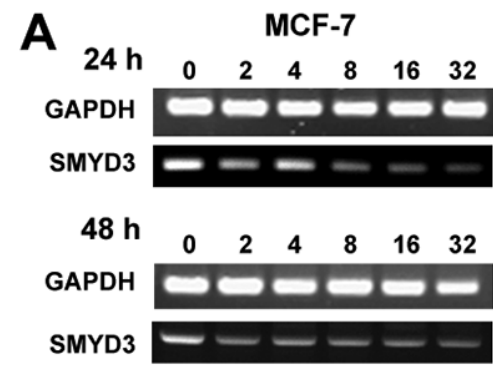

B
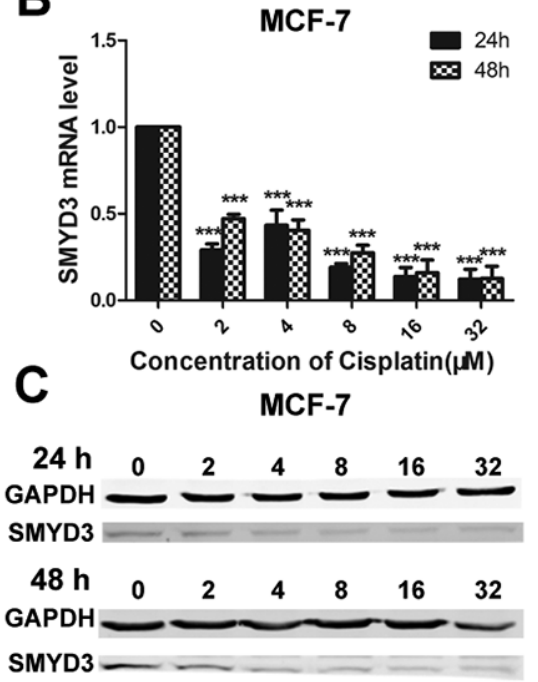

T47D
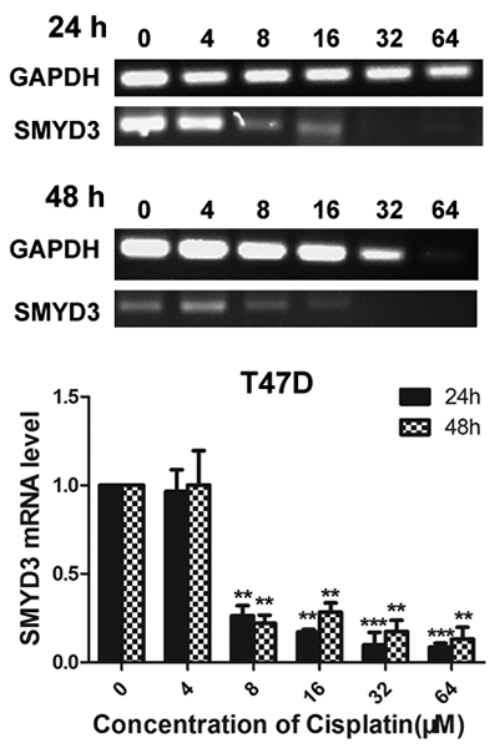

T47D

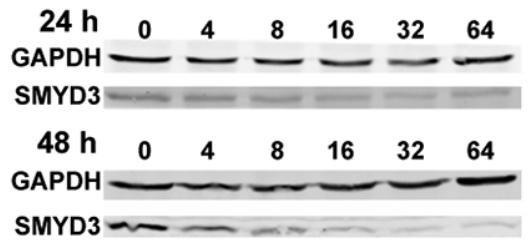

Figure 4. Cisplatin inhibits SMYD3 expression. SMYD3 transcription in cisplatin-treated MCF-7 cells was analyzed by (A) semi-qPCR and (B) reverse transcription-qPCR. (C) SMYD3 expression in cisplatin-treated MCF-7 and T47D cells was analyzed by western blotting. Mean \pm SD $, n=3,{ }^{* *} \mathrm{P}<0.01,{ }^{* * * *} \mathrm{P}<0.001$ vs. $0 \mu \mathrm{M}$ cisplatin. qPCR, quantitative PCR; SMYD3, SET and MYND domain-containing protein 3.

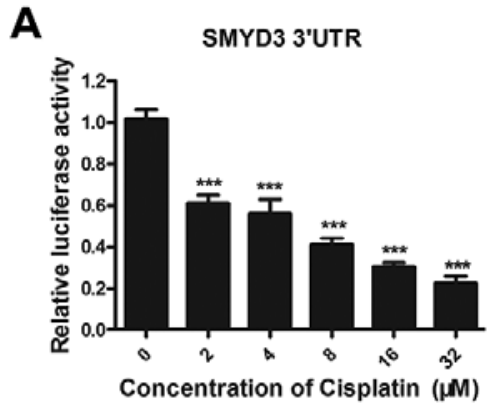

B

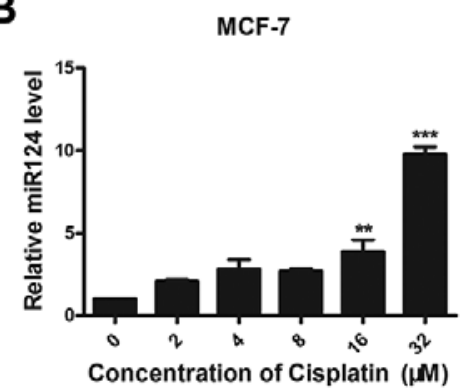

SMYD3 3'UTR

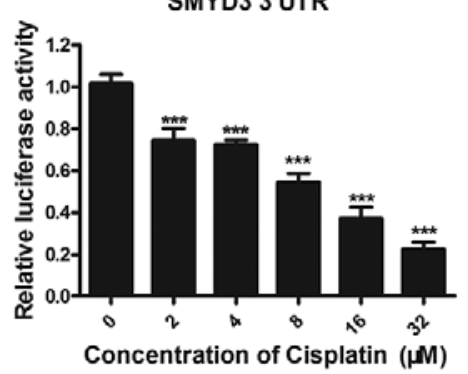

T47D

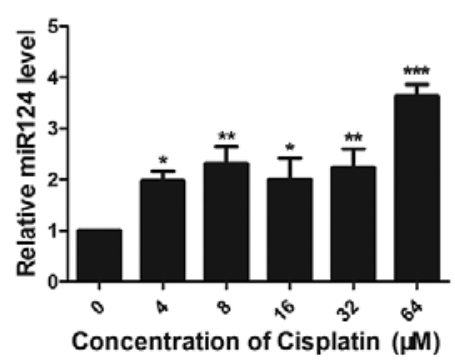

Figure 5. miR-124 may be involved in the cisplatin-mediated SMYD3 expression inhibition. (A) Effect of cisplatin on SMYD3 3'UTR activity assessed by luciferase assays. MCF-7 cells (left panel) and T47D cells (right panel). (B) Effect of cisplatin on miR-124 level. Mean $\pm \mathrm{SD} . \mathrm{n}=3 .{ }^{*} \mathrm{P}<0.05,{ }^{* * *} \mathrm{P}<0.01,{ }^{* * * *} \mathrm{P}<0.001$ vs. $0 \mu \mathrm{M}$ cisplatin. SMYD3, SET and MYND domain-containing protein 3; 3'UTR, 3'-untranslated region.

MAPK family members are also involved in the cisplatin resistance mechanism. The N-terminal kinases of c-JUN and MAPK1 cannot be activated in cisplatin-resistant cells, leading to an inability to initiate the FAS signaling pathway and therefore cell apoptosis (36). In addition, previous studies demonstrated that SMYD3-mediated MAP3K2 
methylation prevents MAP3K 2 from binding to the protein phosphatase 2, inhibiting the effect of this negative regulator on Ras-ERK1/2 signals, which could lead to the development of lung and pancreatic adenocarcinomas $(37,38)$. The results from the present study demonstrated that the combination of SMYD3 knockdown and cisplatin-mediated SMYD3 expression inhibition may promote the mitochondrial membrane potential collapse and subsequently decrease the viability of MCF-7 cells.

In the present study, cisplatin treatment could downregulate SMYD3 expression and inhibit the transcriptional regulatory activity of the SMYD3 3'UTR. Specific interactions between miRNAs and 3'UTRs are known to promote the degradation of target mRNA (31). Furuta et al (21) reported that miR-124 can inhibit the growth of cancer by targeting SMYD3. Furthermore, it has been reported that miR-124 can promote cancer cell sensitivity to cisplatin (22), and the findings of the present study demonstrated that the expression of SMYD3 has a negative regulatory relationship with miR-124. In addition, sulforaphane can enhance the effects of cisplatin by activating miR-124 (39). Our previous study also demonstrated that SMDY3 may have a crucial role in the anti-tumor effect of sulforaphane (15). Taken together, these findings suggested that SMYD3 may impair cell sensitivity to cisplatin, and that miR-124 may serve a crucial role in this process. These results may help future investigation on SMYD3 expression interference by cisplatin, contribute to the development of therapeutic options to circumvent cisplatin resistance and therefore allow cancer treatment.

\section{Acknowledgements}

Not applicable.

\section{Funding}

The current study was supported by the National Natural Science Foundation of China (grant nos. 31470816 and 31300642), the Natural Science Foundation of Tianjin (grant no. 18JCZDJC33800) and the Young Teachers' Innovation Fund of Tianjin University of Science and Technology (grant no. 2016LG06).

\section{Availability of data and materials}

All data generated or analyzed during the present study are included in this published article.

\section{Authors' contributions}

LW, XL, NW, HH and TZ conceived and designed the experiments. LW, MX and CW performed the experiments and analyzed the data, and were major contributors in writing the manuscript. QD, ZM and XC provided technical guidance and participated in data acquisition and analysis. All the authors read and approved the final version of the manuscript.

\section{Ethics approval and consent to participate}

Not applicable.

\section{Patient consent for publication}

Not applicable.

\section{Competing interests}

The authors declare that they have no competing interests.

\section{References}

1. Rosenberg B: Cisplatin: Its history and possible mechanisms of action. In: Prestayko AW, Crooke ST and Carter SK (eds): Cisplatin: Current Status and New Developments. Academic Press, NYC, pp9-21, 1980.

2. Dasari S and Tchounwou PB: Cisplatin in cancer therapy: Molecular mechanisms of action. Eur J Pharmacol 740: 364-378, 2014.

3. Joss RA, Bürki K, Dalquen P, Schatzmann E, Leyvraz S, Cavalli F, Ludwing C, Siegenthaler P, Allberto P, Stahel R, et al: Combination chemotherapy with mitomycin, vindesine, and cisplatin for non-small cell lung cancer association of antitumor activity with initial tumor burden and treatment center. Cancer 65: 2426-2434, 1990.

4. Garutti M, Pelizzari G, Bartoletti M, Malfatti MC, Gerratana L, Tell G and Puglisi F: Platinum salts in patients with breast cancer: A focus on predictive factors. Int J Mol Sci 20: E3390, 2019.

5. Eljack ND, Ma HY, Drucker J, Shen C, Hambley TW, New EJ, Friedrich T and Clarke RJ: Mechanisms of cell uptake and toxicity of the anticancer drug cisplatin. Metallomics 6: 2126-2133, 2014.

6. Li C, Liu M, Yan A, Liu W, Hou J, Cai L and Dong X: ERCC1 and the efficacy of cisplatin in patients with resected non-small cell lung cancer. Tumour Biol 35: 12707-1212, 2014.

7. Cocetta V, Ragazzi E and Montopoli M: Mitochondrial Involvement in Cisplatin Resistance. Int J Mol Sci 20: E3384, 2019.

8. Bao A, Li Y, Tong Y, Zheng $\mathrm{H}, \mathrm{Wu} \mathrm{W}$ and Wei C: 1,25-Dihydroxyvitamin $\mathrm{D}_{3}$ and cisplatin synergistically induce apoptosis and cell cycle arrest in gastric cancer cells. Int $\mathrm{J}$ Mol Med 33: 1177-1184, 2014.

9. Hrzenjak A, Fischer C, Leithner K, Wohlkoenig C, Quehenberger F, Olschewski A and Olschewski H: 838: Panobinostat reduces hypoxia-related cisplatin resistance of non-small cell lung carcinoma cells via HIF-1alpha destabilization. Eur J Cancer 50 (Suppl 5): S203-S204, 2014.

10. Latifi A, Abubaker K, Castrechini N, Ward AC, Liongue C, Dobill F, Kumar J, Thompson EW, Quinn MA, Findlay JK and Ahmed N: Cisplatin treatment of primary and metastatic epithelial ovarian carcinomas generates residual cells with mesenchymal stem cell-like profile. J Cell Biochem 112: 2850-2864, 2011.

11. Sahu BD, Kalvala AK, Koneru M, Mahesh Kumar J, Kuncha M, Rachamalla SS and Sistla R: Ameliorative effect of fisetin on cisplatin-induced nephrotoxicity in rats via modulation of NF- $\kappa \mathrm{B}$ activation and antioxidant defence. PLoS One 9: e105070, 2014.

12. Liao HY, Wang GP, Gu LJ, Huang SH, Chen XL, Li Y and Cai SW: HIF-1 $\alpha$ siRNA and cisplatin in combination suppress tumor growth in a nude mice model of esophageal squamous cell carcinoma. Asian Pac J Cancer Prev 13: 473-477, 2012.

13. Hamamoto R, Silva FP, Tsuge M, Nishidate T, Katagiri T, Nakamura Y and Furukawa Y: Enhanced SMYD3 expression is essential for the growth of breast cancer cells. Cancer Sci 97: 113-118, 2006.

14. Zou JN, Wang SZ, Yang JS, Luo XG, Xie JH and Xi T: Knockdown of SMYD3 by RNA interference down-regulates c-Met expression and inhibits cells migration and invasion induced by HGF. Cancer Lett 280: 78-85, 2009.

15. Wang L, Wang QT, Liu YP, Dong QQ, Hu HJ, Miao Z, Li S, Liu Y, Zhou H, Zhang TC, et al: ATM signaling pathway is implicated in the SMYD3-mediated proliferation and migration of gastric cancer cells. J Gastric Cancer 17: 295-305, 2017.

16. Hu P, Chu GC, Zhu G, Yang H, Luthringer D, Prins G, Habib F, Wang Y, Wang R, Chung LW and Zhau HE: Multiplexed quantum dot labeling of activated c-Met signaling in castration-resistant human prostate cancer. PLoS One 6: e28670, 2011.

17. Liu C, Fang X, Ge Z, Jalink M, Kyo S, Bjorkholm M, Gruber A, Sjoberg $\mathrm{J}$ and $\mathrm{Xu} \mathrm{D}$ : The telomerase reverse transcriptase (hTERT) gene is a direct target of the histone methyltransferase SMYD3. Cancer Res 67: 2626-2631, 2007. 
18. Hassan KA, Wang L, O'Dowd P, Kim G, Korkaya H, Davis A, Liu S, Kalemkerian GP and Wicha MS: Abstract 3309: Blocking the Notch pathway inhibits the epithelial-mesenchymal transition (EMT) status in lung cancer and alters chemoresistance. Cancer Res 72: 3309-3309, 2012.

19. Chen YJ, Tsai CH, Wang PY and Teng SC: SMYD3 promotes homologous recombination via regulation of $\mathrm{H} 3 \mathrm{~K} 4$-mediated gene expression. Sci Rep 7: 3842, 2017.

20. Zeng B, Li Z, Chen R, Guo N, Zhou J, Zhou Q, Lin W, Cheng D, Liao Q, Zheng L and Gong Y: Epigenetic regulation of miR-124 by hepatitis $\mathrm{C}$ virus core protein promotes migration and invasion of intrahepatic cholangiocarcinoma cells by targeting SMYD3. FEBS Lett 586: 3271-3278, 2012.

21. Furuta M, Kozaki KI, Tanaka S, Arii S, Imoto I and Inazawa J: miR-124 and miR-203 are epigenetically silenced tumor-suppressive microRNAs in hepatocellular carcinoma. Carcinogenesis 31: 766-776, 2010

22. Xiang S, Fanyi K, Zhenfeng Z, Mingming R, Qingjun M, Yanguang LI and Zhen S: miR-124 and miR-142 enhance cisplatin sensitivity of non-small cell lung cancer cells through repressing autophagy via directly targeting SIRT1. RSC Adv 9: 5234-5243, 2019.

23. Luo XG, Ding Y, Zhou QF, Ye L, Wang SZ and Xi T: SET and MYND domain-containing protein 3 decreases sensitivity to dexamethasone and stimulates cell adhesion and migration in NIH3T3 cells. J Biosci Bioeng 103: 444-450, 2007.

24. Livak K and Schmittgen T: Analysis of relative gene expression data using real-time quantitative PCR and the 2(-Delta Delta C(T)) method. Methods 25: 402-408, 2001.

25. Dandekar DH, Kumar M, Ladha JS, Ganesh KN and Mitra D: A quantitative method for normalization of transfection efficiency using enhanced green fluorescent protein. Anal Biochem 342: 341-344, 2005

26. Del Bello B, Valentini MA, Zunino F, Comporti M and Maellaro E: Cleavage of Bcl-2 in oxidant- and cisplatin-induced apoptosis of human melanoma cells. Oncogene 20: 4591-4595, 2001.

27. Isonishi S, Saitou M, Yasuda M, Ochiai K and Tanaka T: Enhancement of sensitivity to cisplatin by orobol is associated with increased mitochondrial cytochrome $\mathrm{c}$ release in human ovarian carcinoma cells. Gynecol Oncol 90: 413-420, 2003.

28. Johnson LV, Walsh ML and Chen LB: Localization of mitochondria in living cells with rhodamine 123. Proc Natl Acad Sci USA 77: 990-994, 1980.
29. Sarris ME, Moulos P, Haroniti A, Giakountis A and Talianidis I: Smyd 3 is a transcriptional potentiator of multiple cancer-promoting genes and required for liver and colon cancer development. Cancer Cell 29: 354-366, 2016.

30. Fei X, Ma Y, Liu X and Meng Z: Overexpression of SMYD3 is predictive of unfavorable prognosis in hepatocellular carcinoma. Tohoku J Exp Med 243: 219-226, 2017.

31. Fang $Z$ and Rajewsky N: The impact of miRNA target sites in coding sequences and in 3'UTRs. PLoS One 6: e18067, 2011.

32. Bottino C, Peserico A, Simone C and Caretti G: SMYD3: An oncogenic driver targeting epigenetic regulation and signaling pathways. Cancers (Basel) 12: E142, 2020.

33. Sprowl JA, Lancaster CS, Pabla N, Hermann E, Kosloske AM, Gibson AA, Li L, Zeeh D, Schlatter E, Janke LJ, et al: Cisplatin-induced renal injury is independently mediated by OCT2 and p53. Clin Cancer Res 20: 4026-4035, 2014.

34. Abedini MR, Muller EJ, Brun J, Bergeron R, Gray DA and Tsang BK: Cisplatin induces p53-dependent FLICE-like inhibitory protein ubiquitination in ovarian cancer cells. Cancer Res 68: 4511-4517, 2008.

35. Dai B, Wan W, Zhang P, Zhang Y, Pan C, Meng G, Xiao X, Wu Z, Jia W, Zhang J and Zhang L: SET and MYND domain-containing protein 3 is overexpressed in human glioma and contributes to tumorigenicity. Oncol Rep 34: 2722-2730, 2015.

36. Brozovic A, Fritz G, Christmann M,Zisowsky J, Jaehde U, Osmak M and Kaina B: Long-term activation of SAPK/JNK, p38 kinase and fas-L expression by cisplatin is attenuated in human carcinoma cells that acquired drug resistance. Int J Cancer 112: 974-985, 2004.

37. Colon-Bolea $\mathrm{P}$ and Crespo P: Lysine methylation in cancer: SMYD3-MAP3K2 teaches us new lessons in the Ras-ERK pathway. Bioessays 36: 1162-1169, 2014.

38. Riera TV, Wigle TJ, Gureasko J, Boriack-Sjodin PA and Copeland RA: Abstract 2144: Kinetic mechanism of the lysine methyltransferase SMYD3 using MAP3K2 protein substrate. Cancer Res 75 (15 Suppl): S2144, 2015.

39. Wang X, Li Y, Dai Y, Liu Q, Ning S, Liu J, Shen Z, Zhu D, Jiang F, Zhang J and Li Z: Sulforaphane improves chemotherapy efficacy by targeting cancer stem cell-like properties via the miR-124/IL-6R/STAT3 axis. Sci Rep 6: 36796, 2016.

(i) () $९$ This work is licensed under a Creative Commons

COY NC ND Attribution-NonCommercial-NoDerivatives 4.0 International (CC BY-NC-ND 4.0) License. 